\title{
Directional Change Issues in Multivariable State-feedback Control
}

\author{
Dariusz Horla \\ Poznan University of Technology, \\ Institute of Control and Information Engineering, \\ Department of Control and Robotics \\ ul. Piotrowo 3a, 60-965 Poznan \\ Poland
}

\section{Introduction}

Control limits are ubiquitous in real world, in any application, thus taking them into consideration is of prime importance if one aims to achieve high performance of the control system. One can abide constraints by means of two approaches - the first case is to impose constraints directly at the design of the controllerwhat usually leads to problemswith obtaining explicit forms (or closed-form expressions) of control laws, apart from very simple cases, e.g. quadratic performance indexes. The other approach is based on assuming the system is linear and having imposed constraints on the controller output (designed for unconstrained case - bymeans of optimisation, using Diophantine equations, etc) one has to introduce necessary amendments to the control system because of, possibly, active constraints (Horla, 2004a; Horla, 2007d; Öhr, 2003; Peng et al., 1998).

When internal controller states do not correspond to the actual signals present in the control systems because of constraints, or in general - nonlinearity at controller output, then such a situation is referred in the literature as windup phenomenon (Doná et al., 2000; Horla, 2004a; Öhr, 2003). It is obvious that due to not taking control signal constraints into account during the controller design stage, one can expect inferior performance because of infeasibility of computed control signals.

Many methods of anti-windup compensation are known from the single-input single-output

\& framework, but a few work well enough in the case of multivariable systems (Horla, 2004a; Horla, 2004b; Horla, 2006a; Horla, 2006b; Horla, 2006c; Horla, 2007a; Horla, 2007b; Horla, 2007c; Horla, 2007d; Öhr, 2003; Peng et al., 1998; Walgama \& Sternby, 1993). For multivariable systems have additional feature - windup phenomenon is tightly connected of with directional change phenomenon in the control vector due to different implementations of constraints, affecting in this way the direction of the computed control vector. Even for a simple amplitude-constrained case, the constrained control vector $\underline{u}_{t}$ may have a different direction than a computed control vector $\underline{v}_{t}$. The situation is even more complicated for amplitude and rate-constrained system, where for additional requirements, e.g. keeping constant direction, there may be no appropriate control action to be taken (Horla, 2007d).

Source: New Approaches in Automation and Robotics, Book edited by: Harald Aschemann, ISBN 978-3-902613-26-4, pp. 392, May 2008, I-Tech Education and Publishing, Vienna, Austria 
Apart from windup and directional change phenomena one can expect to obtain inferior performance because of problems with (dynamic) decoupling, especially when the plant does not have equal numbers of control signals and output signals (Albertos \& Sala, 2004; Maciejowski, 1989). In such a case, control direction corresponds not only to input principal directions (or maximal directional gain of the transfer function matrix), but also to the degree of decoupling, and by altering it one achieve better decoupling (though not in all cases).

Directional change has been discussed in (Öhr, 2003; Walgama and Sternby, 1993), where the first description of the problemwas given, connections in between anti-windup compensation and directional change has been made. A review of multivariable antiwindup compensators has been included in (Horla, 2007b; Peng et al., 1998; Walgama \& Sternby, 1993) with basic analysis of the topic.

Windup phenomenon (thus decoupling and directional change) are tightly connected with industrial applications are crucial when control laws are to be applied. Many papers treated application of anti-windup compensation (AWC) in areas as motor drives, paper machine headbox or hydraulic drives control. But they lack in understanding what is the connection in between directional change and AWC.

To recapitulate, when control limits are taken into consideration, the presence of windup phenomenon requires certain actions to compensate it, i.e., to retrieve the correspondence of the internal controller states with its (vector) output. Heuristic modifications feeding back to the controller the portion of controls changed by nonlinearity are performed by a posteriori antiwindup compensators and a priori AWCs enable windup phenomenon avoidance (i.e., a priori compensation) by generating feasible control actions only (Doná et al., 2000; Horla, 2006a; Horla, 2006b).

Having avoided generation of infeasible control actions one avoids windup phenomenon in a priori manner, and implicitly eliminates windup phenomenon that would inevitably take place had control limits not been taken into consideration first.

The chapter aims to focus on directional change issues (and, simultaneously, anti-windup compensation) in multivariable state-feedback controller with a priori anti-windup compensator for systems given in state-space form. The problem is presented through the framework of linear matrix inequalities (LMIs). Imposing only amplitude constraints on the control vector results in LMI conditions, but taking rate constraints into consideration results in nonsymmetric matrix inequality, that is transformed into LMI by making certain assumptions, as in (Horla, 2007a).

\section{Control vector constraints and directional change}

Let us suppose that amplitude constraints are imposed on the input signals of two-input plant. Depending on the method of imposing constraints one can observe directional change, illustrated in Fig. 1a in the case of cut-off saturation that is not present when saturation is performed according to imposed constraints (dashed lines) with constant direction, Fig. 1b.

The situation is more complicated when rate constraints are taken into consideration. Let $\mathscr{D}_{\alpha}$ denote the set of all feasible control vectors due to amplitude constraints and $\mathscr{D}_{\boldsymbol{\beta}}$ denote the set of all feasible control vectors due to rate constraints. If $\mathscr{D}_{\alpha} \cap \mathscr{D}_{\beta} \neq 0$ than constrained 
input vector is feasible. The aim is to constrain the control vector so that as much of its primal information is kept with minimum directional change (Horla, 2007c).

Let the computed control vector violate amplitude constraints and have the property that its amplitude-constrained companion does not violate rate constraints, i.e. $\underline{u}_{t} \in \mathscr{D}_{\boldsymbol{\beta}}$ (see Fig. 2a). The necessary condition here for control direction to be preserved is as above, for such a case only two sets: a point $\left(\underline{u}_{1, t} \underline{u}_{2}, t\right)$ in the plane and $\mathscr{D}_{\beta}$ have a common part.
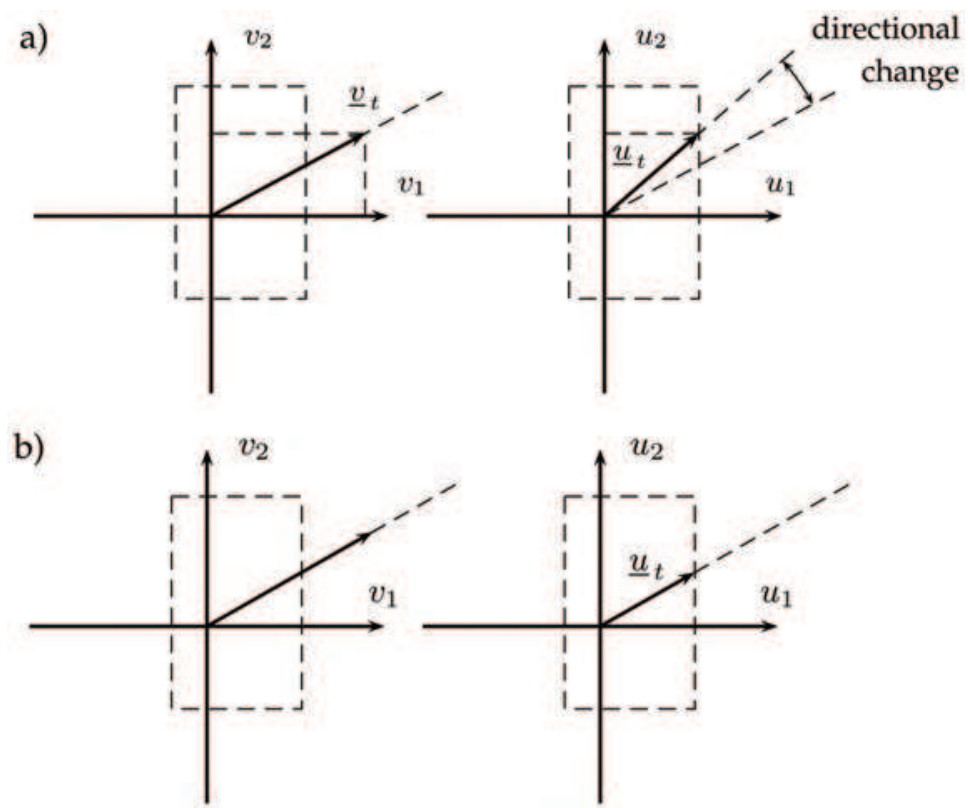

Fig. 1. a) direction-changing, b) direction-preserving saturation (left: control vector before saturation, right: after saturation)

When the point on the end of direction-preserved, amplitude-constrained, computed control vector and $\mathscr{D}_{\boldsymbol{\beta}}$ do not have a common part, than it is impossible to generate feasible control actions with both amplitude and rate constraints imposed so that direction of the computed control vector is sustained. This is depicted in Figure $2 b$, where the only constrained control vector lies ",as close as possible" to the computed control vector satisfies $\underline{u}_{\mathrm{t}} \in \mathscr{D}_{\alpha} \cap \mathscr{D}_{\boldsymbol{\beta}}$ and $\underline{u}_{\mathrm{t}}$ $\in \mathrm{b}\left(\mathscr{D}_{\beta}\right)$ (boundary of $\mathscr{D}_{\beta}$ ). When rate constraints are violated, one has to treat them either as secondary constraints (to be omitted) or introduce soft rate constraints instead of hard rate constraints (Maciejowski, 1989).

\section{Directional change phenomenon, an example}

Let two-input two-output system be not coupled and both loops be driven by separate controllers (with no cross-coupling). The systemoutput $y_{\mathrm{t}}$ is to track reference vector comprising two sinusoid waves, what corresponds to drawing a circular shape in the $\left(y_{1}, y_{2}\right)$ plane. 


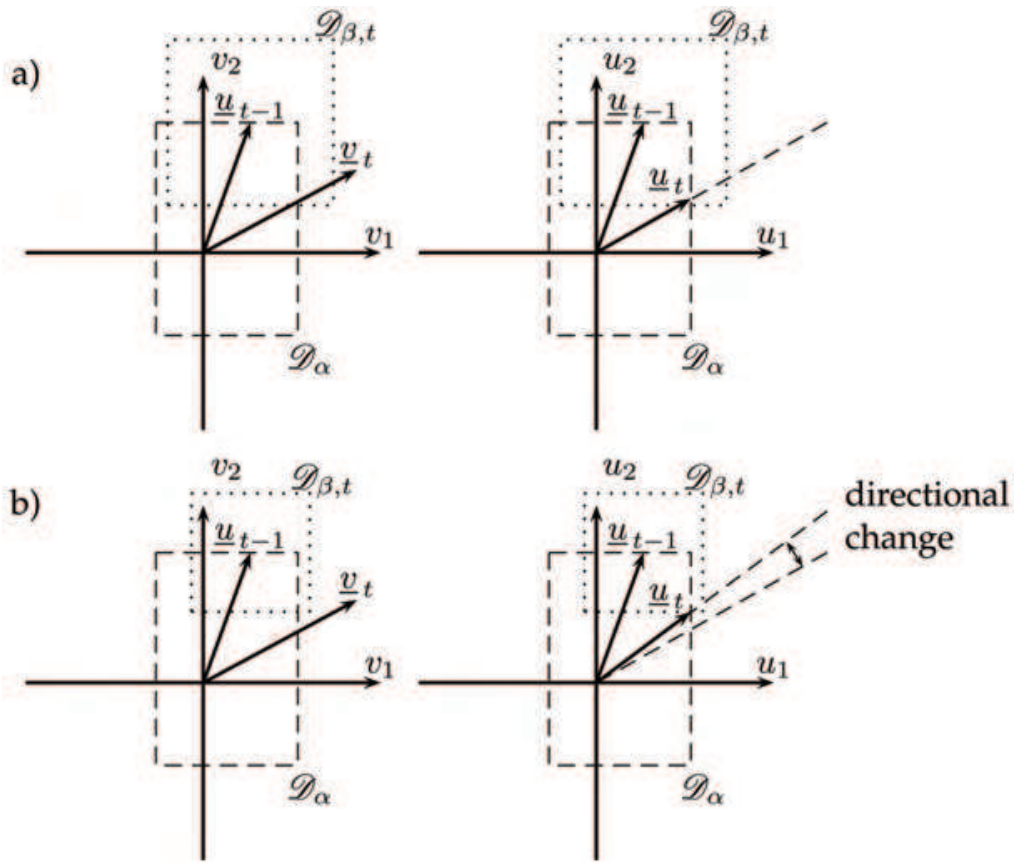

Fig. 2. a) direction-preserved saturation, b) saturation with directional change
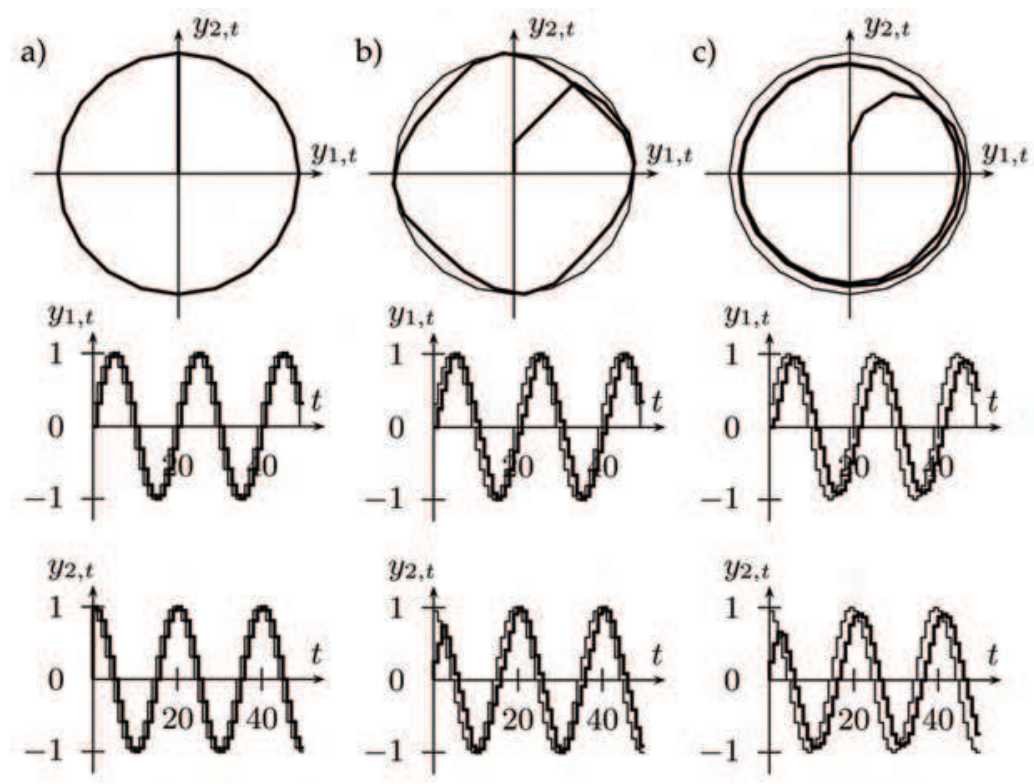

Fig. 3. a) unconstrained system, b) cut-off saturation, c) direction-preserving saturation 
As it can be seen in the Fig. 3a, the unconstrained system performs best, whereas in the case of cut-off saturation imposed on both elements of control vector (Fig. 3b) the tracking performance is poor. This is because of directional change in controls that changes proportions between its components. In the application for, e.g., shape-cutting, performance of the system from Fig. 3c (direction-preserving saturation) is superior. Furthermore, in order to achieve such a performance the system must be perfectly decoupled at all times (Horla, 2007b; Öhr, 2003).
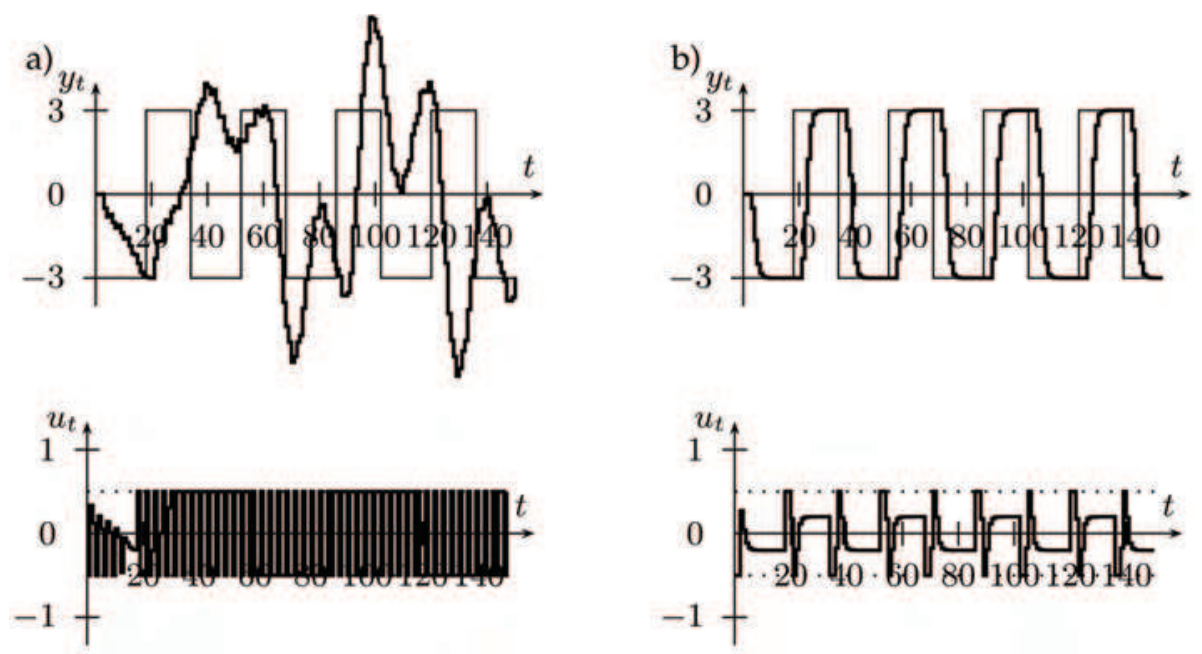

Fig. 4. results from closed-loop system a) without AWC, b) with AWC

\section{Anti-windup compensation, an example}

An example action of AWC is shown in Figure 4, where for hard constraints imposed on the control signal and pole-placement controller it is impossible to ensure tracking properties if windup phenomenon is left uncompensated. On the other hand, by performing compensation, the control signal is desaturated and is not prone to consecutive resaturations, operating in a period of time in a linear zone.

\section{How to understand windup phenomenon in multivariable systems - literature remarks}

The problem of windup phenomenon in multivariable systems with its connection to directional change in controls has rarely been addressed in the literature. The only valuable remark concerning directional change is in (Walgama and Sternby, 1993):

Solving the windup phenomenon problem does not mean that constrained control vector is of the same direction as computed control vector.

On the other hand, avoiding directional change in control enables one to avoid windup phenomenon. In further parts of this chapter, it will be described where the latter description holds. 


\section{Considered plant model}

The directional change issues are discussed for state-feedback control law that has been derived for shifted-input $\underline{u}_{t}^{s} \in \mathscr{R}^{m}$ and shifted-output $\underline{y}_{t}^{s} \in \mathscr{R}^{p}$ plant in the CARMA structure, taking into account the offset resulting from the current set-point vector for plants without integration (in a steady-state)

$$
\boldsymbol{A}\left(q^{-1}\right) \underline{y}_{t}^{s}=\boldsymbol{B}\left(q^{-1}\right) \underline{u}_{t-d}^{s},
$$

represented in state-space representation for non-shifted (original) inputs and outputs:

$$
\begin{gathered}
\underline{x}_{t+1}=\boldsymbol{A} \underline{x}_{t}+\boldsymbol{B} \underline{u}_{t-d}, \\
\underline{y}_{t}=\boldsymbol{C} \underline{x}_{t},
\end{gathered}
$$

with

$$
\begin{gathered}
\boldsymbol{A}=\left[\begin{array}{cccc}
-\boldsymbol{A}_{1} & \boldsymbol{I} & \cdots & \mathbf{0} \\
\cdots & \ldots & \cdots & \mathbf{0} \\
-\boldsymbol{A}_{n-1} & \cdots & \cdots & \boldsymbol{I} \\
-\boldsymbol{A}_{n} & \cdots & \cdots & \mathbf{0}
\end{array}\right] \\
\boldsymbol{B}=\left[\boldsymbol{B}_{1}^{T}, \ldots, \boldsymbol{B}_{n}^{T}\right]^{T}, \\
\boldsymbol{C}=[\boldsymbol{I}, \mathbf{0}, \ldots, \mathbf{0}]^{T}, \\
\boldsymbol{A}\left(q^{-1}\right)=\boldsymbol{I}+\boldsymbol{A}_{1} q^{-1}+\cdots+\boldsymbol{A}_{n} q^{-n}, \\
\boldsymbol{B}\left(q^{-1}\right)=\boldsymbol{B}_{1}+\boldsymbol{B}_{2} q^{-1}+\cdots+\boldsymbol{B}_{n-1} q^{-n} .
\end{gathered}
$$

The aim of state-feedback controller is to track a given reference vector $\underline{r}_{\mathrm{t}} \in \mathscr{R}^{p}$ with plant output vector $\underline{y}{ }_{t}^{s}$ minimising certain performance index. The offset previouslymentioned:

$$
\begin{aligned}
& \underline{x}_{t}^{s}=\underline{x}_{t}+\underline{x}_{\infty}, \\
& \underline{u}_{t}^{s}=\underline{u}_{t}+\underline{u}_{\infty}, \\
& \underline{y}_{t}^{s}=\underline{y}_{t}+\underline{y}_{\infty},
\end{aligned}
$$

where for plants without integral terms

$$
\underline{x}_{\infty}=(\boldsymbol{I}-\boldsymbol{A})^{-1} \boldsymbol{B} \underline{u}_{\infty},
$$




$$
\underline{y}_{\infty}=\underline{r}_{t},
$$

with $\underline{u}_{\infty} \in \mathscr{R}^{m}$ being the control vector value assuring tracking in steady state, i.e.

$$
\boldsymbol{A}(1) \underline{r}_{t}=\boldsymbol{B}(1) \underline{u}_{\infty} .
$$

For plants comprising integral terms there is no need to shift states because $\underline{u}_{\infty} \neq \underline{0}$ holds. In the case of $m \neq p$, the control vector offset results from (14) by means of pseudoinverse.

The considered state-feedback controller enables one to constrain the part of $\underline{u}_{\mathrm{t}}$ which is related to the answer of the plant to non-zero initial conditions at instant $\underline{t}$, which subsequently corresponds to the current values of reference vector. In other words, it enables constraining the absolute value of the elements of $\underline{u}_{t}^{s}$ that are above absolute value of $\underline{u}_{\infty}$.

In order to fully analyze the directional change phenomenon interplay with AWC, rate vector constraints $\Delta \underline{u}_{\mathrm{t}}=\underline{u}_{\mathrm{t}}-\underline{u}_{\mathrm{t}-1}$ are also to be taken into account here (Horla, 2007c; Horla, 2007a).

\section{State-feedback controller}

At each time instant the optimal state-feedback matrix is generated (Horla, 2006b)

$$
\boldsymbol{F}_{t}^{*}: J_{t}\left(\boldsymbol{F}_{t}^{*}\right)=\inf _{\boldsymbol{F}_{t} \in \mathscr{D}\left(J_{t}\right)}\left\{J_{t}\left(\boldsymbol{F}_{t}\right)\right\}
$$

assuming that $\underline{r}_{\mathrm{t}}$ is of constant value $\underline{r}_{,} \underline{u}_{\mathrm{t}}=\boldsymbol{F}_{\mathrm{t}} \underline{x}_{\mathrm{t}}$, state vector is perfectly measured and constraints are imposed on the control vector. The set $\mathscr{D}$ denotes all $\boldsymbol{F}_{\mathrm{s}}$, for which the performance index $J_{\mathrm{t}}$ is of finite value.

At each time instant the optimisation procedure of $\boldsymbol{F}_{t}^{\star}$ is performed for current values of reference vector, and at the next instant the procedure is repeated again.

The performance index

$$
J_{t}=\sum_{i=t}^{\infty}\left(\left\|\underline{x}_{i}\right\|_{Q_{1}}^{2}+\left\|\underline{u}_{i}\right\|_{R_{1}}^{2}\right),
$$

is a quadratic function and is minimised subject to $\boldsymbol{F}_{\mathrm{t}}$, with state-feedback control law $\underline{u}_{\mathrm{t}}=\boldsymbol{F}_{\mathrm{t}} \underline{x}_{\mathrm{t}}$. As it has been already said, plant state is fully accessible (if not, one can perform estimation on the basis of the separation theorem).

In order to minimise the performance index (16), its upper bound is to be found. Let the following Lyapunov function be given

$$
V\left(\underline{x}_{t}\right)=\underline{x}_{t}^{T} \boldsymbol{P}_{x_{t}},
$$

with positive definite $\boldsymbol{P}>0$, and $\mathrm{V}(\underline{0})=0$. Having assumed that at each time instant $t$ holds

$$
V\left(\underline{x}_{t+1}\right)-V\left(\underline{x}_{t}\right) \leqslant-\left(\underline{x}_{t}^{T} \boldsymbol{Q}_{1} \underline{x}_{t}+\underline{u}_{t}^{T} \boldsymbol{R}_{1} \underline{u}_{t}\right),
$$


which left - and right-hand side sum from $t=t$ to $t=\infty$ satisfies

$$
-V\left(\underline{x}_{t}\right) \leqslant-J_{t},
$$

the performance index (16) is bounded from above with

$$
J_{t} \leqslant V\left(\underline{x}_{t}\right),
$$

from where using (17) one obtains

$$
J_{t} \leqslant \underline{x}_{t}^{T} \boldsymbol{P}_{\underline{x}_{t}},
$$

Minimisation of the quadratic form $\underline{x}_{t}^{T} \boldsymbol{P} \underline{x}_{t}$, with $\boldsymbol{P}>0$, on the basis of Schur complement for $\underline{x}_{t}^{T} \boldsymbol{P} \underline{x}_{\mathrm{t}} \leq \gamma$ is equivalent to linear matrix inequality (LMI) (Boyd et al., 1994; Boyd and Vandenberghe, 2004)

$$
\begin{array}{ll}
\min \gamma & \\
\text { s.t. } & {\left[\begin{array}{cc}
1 & \star \\
\underline{x}_{t} & \boldsymbol{Q}
\end{array}\right] \geqslant 0}
\end{array}
$$

for $\gamma>0$ and $Q=\gamma \boldsymbol{P}^{-1}$.

Having substituted (17) into (18)

$$
\underline{x}_{t+1}^{T} \boldsymbol{P} \underline{x}_{t+1}-\underline{x}_{t}^{T} \boldsymbol{P} \underline{x}_{t} \leqslant-\left(\underline{x}_{t}^{T} \boldsymbol{Q}_{1} \underline{x}_{t}+\underline{u}_{t}^{T} \boldsymbol{R}_{1} \underline{u}_{t}\right),
$$

using (2), and putting $\underline{u}_{\mathrm{t}}=F \underline{x}_{\mathrm{t}}$ one can write

$$
\left((\boldsymbol{A}+\boldsymbol{B} \boldsymbol{F}) \underline{x}_{t}\right)^{T} \boldsymbol{P}\left((\boldsymbol{A}+\boldsymbol{B} \boldsymbol{F}) \underline{x}_{t}\right)-\underline{x}_{t}^{T} \boldsymbol{P} \underline{x}_{t}+\underline{x}_{t}^{T} \boldsymbol{Q}_{1} \underline{x}_{t}+\left(\boldsymbol{F} \underline{x}_{t}\right)^{T} \boldsymbol{R}_{1}\left(\boldsymbol{F} \underline{x}_{t}\right) \leqslant 0
$$

which negative-definiteness is equivalent to

$$
(\boldsymbol{A}+\boldsymbol{B F})^{T} \boldsymbol{P}(\boldsymbol{A}+\boldsymbol{B F})-\boldsymbol{P}+\boldsymbol{F}^{T} \boldsymbol{R}_{1} \boldsymbol{F}+\boldsymbol{Q}_{1} \leqslant 0 .
$$

Putting $\boldsymbol{P}=\gamma \boldsymbol{Q}^{-1}$ post- and pre-multiplying with $\boldsymbol{Q}^{\mathrm{T}}$ and $\boldsymbol{Q}$, and putting $\boldsymbol{Y}=\boldsymbol{F} \boldsymbol{Q}$, the inequality can be rewritten as

$$
Q-\frac{1}{\gamma} \boldsymbol{Y}^{T} R_{1} \boldsymbol{Y}-\frac{1}{\gamma} \boldsymbol{Q}^{T} \boldsymbol{Q}_{1} \boldsymbol{Q}-(A Q+B Y)^{T} \boldsymbol{Q}^{-1}(A \boldsymbol{Q}+B \boldsymbol{Y}) \geqslant 0 .
$$

Applying the Schur complement again one obtains

$$
\begin{aligned}
& {\left[\begin{array}{cc}
Q-\frac{1}{\gamma} \boldsymbol{Y}^{T} \boldsymbol{R}_{1} \boldsymbol{Y}-\frac{1}{\gamma} \boldsymbol{Q}^{T} \boldsymbol{Q}_{1} \boldsymbol{Q} & \star \\
\boldsymbol{A} \boldsymbol{Q}+\boldsymbol{B} \boldsymbol{Y} & \boldsymbol{Q}
\end{array}\right] \geqslant 0,} \\
& {\left[\begin{array}{cc}
\boldsymbol{Q} & \star \\
\boldsymbol{A} \boldsymbol{Q}+\boldsymbol{B} \boldsymbol{Y} & \boldsymbol{Q}
\end{array}\right]-\frac{1}{\gamma}\left[\begin{array}{cc}
\boldsymbol{Q}^{T} \boldsymbol{Q}_{1} \boldsymbol{Q}+\boldsymbol{Y}^{T} \boldsymbol{R}_{1} \boldsymbol{Y} & \star \\
\mathbf{0} & \mathbf{0}
\end{array}\right]=} \\
& =\left[\begin{array}{cc}
Q & \star \\
A Q+B Y & Q
\end{array}\right]-\frac{1}{\gamma}\left[\begin{array}{cc}
Q^{T} Q_{1}^{1 / 2} & Y^{T} R_{1}^{1 / 2} \\
0 & 0
\end{array}\right]\left[\begin{array}{ccc}
Q_{1}^{1 / 2} Q & 0 \\
R_{1}^{1 / 2} Y & 0
\end{array}\right]=
\end{aligned}
$$

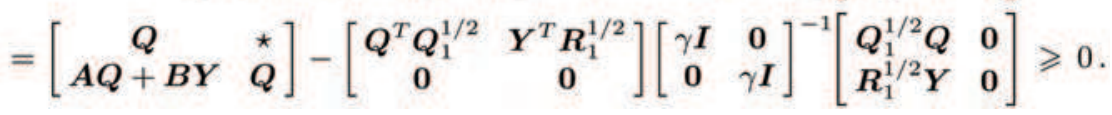


Having applied the Schur complement again, the optimal control law minimising (17) is given as

$$
\underline{u}_{t}=\boldsymbol{F}_{t} \underline{x}_{t},
$$

with (time index has been omitted)

$$
\boldsymbol{F}=\boldsymbol{Y} \boldsymbol{Q}^{-1}
$$

where $Q>0$ and $\boldsymbol{Y}$ are solutions of

$$
\begin{gathered}
\min \quad \gamma \\
\text { s.t. }\left[\begin{array}{cc}
1 & \star \\
\underline{x}_{t} & \boldsymbol{Q}
\end{array}\right] \geqslant 0, \\
{\left[\begin{array}{cccc}
\boldsymbol{Q} & \star & \star & \star \\
\boldsymbol{A} \boldsymbol{Q}+\boldsymbol{B} \boldsymbol{Y} & \boldsymbol{Q} & \star & \star \\
\boldsymbol{Q}_{1}^{1 / 2} \boldsymbol{Q} & \mathbf{0} & \gamma \boldsymbol{I} & \star \\
\boldsymbol{R}_{1}^{1 / 2} \boldsymbol{Y} & \mathbf{0} & \mathbf{0} & \gamma \boldsymbol{I}
\end{array}\right] \geqslant 0 .}
\end{gathered}
$$

One can also take symmetrical control vector constraints into account. Let

$$
\left\|\underline{u}_{t}\right\|_{\max }=\max _{i}\left|u_{t, i}\right|,
$$

$1 \leq i \leq m$, with $\underline{u}_{t} \in \mathscr{R}^{m}$. If there exist $\gamma, Q, Y$, satisfying (30)-(32), than there holds

$$
\left\|\underline{x}_{t}\right\| \leqslant \rho,
$$

and every state $\left\|\underline{x}_{t+j}\right\| \leqslant \rho(j>0)$ lies inside the invariant ellipsoid $\varepsilon_{P}=\left\{\underline{z}:\|\underline{z}\|_{P} \leqslant \rho\right\}$, with $\rho=\left\|\underline{x}_{t}\right\|^{P}$ for invariant reference vector (Horla, 2007a; Horla, 2006b).

Imposing symmetrical constraints on amplitudes of elements of $\underline{u}_{\mathrm{t}}$ is equivalent to imposing constraints on

$$
\left\|\underline{u}_{t}\right\|_{\max }=\left\|\boldsymbol{F} \underline{x}_{t}\right\|_{\max }=\left\|\boldsymbol{Y} \boldsymbol{Q}^{-1} \underline{x}_{t}\right\|_{\max } \leqslant \max _{\underline{x} \in \varepsilon} \| \boldsymbol{Y} \boldsymbol{Q}^{-1} \underline{x}_{\max },
$$

or

$$
\left\|\underline{u}_{t}\right\|_{\max } \leqslant \max _{\underline{x} \in \varepsilon}\left\|\boldsymbol{Y} \boldsymbol{Q}^{-1} \underline{x}\right\|_{\max }=\max _{i}\left(\boldsymbol{Y} \boldsymbol{Q}^{-1} \boldsymbol{Y}^{T}\right)_{i i} .
$$

Than imposing different constraints on each of the elements of control vector is equivalent to LMI

$$
\left[\begin{array}{ll}
\boldsymbol{\Lambda}_{\alpha} & \star \\
\boldsymbol{Y}^{T} & \boldsymbol{Q}
\end{array}\right] \geqslant 0,
$$

Where $\Lambda_{\alpha}=\operatorname{diag}\left\{\alpha_{1}^{2}, \ldots, \alpha_{m}^{2}\right\}$ is a diagonal matrix comprising squares of amplitude constraints of $u_{1, \mathrm{t}}, \ldots, u_{\mathrm{m}, \mathrm{t}}$ on its diagonal. 
The amplitudes of elements of the control vector $\underline{u}_{\mathrm{t}}$ are constrained if the above LMI is added to the set of LMIs (30)-(32).

The presented state-feedback control law stabilises the closed-loop system, guaranteed by the existence of invariant ellipsoid describing $V\left(\underline{x}_{t}\right)$ with fully known plant and constant reference vector.

In a similar manner, one can impose rate of changes constraints. If $\underline{x}_{\mathrm{t}} \approx \underline{x}_{\mathrm{t}-1}$ is a state-vector of approximately constant value, or in a steady-state, than

$$
\Delta \underline{u}_{t}=\boldsymbol{F} \underline{x}_{t}-\boldsymbol{F}_{t-1} \underline{x}_{t-1}=\left(\boldsymbol{F}-\boldsymbol{F}_{t-1}\right) \underline{x}_{t},
$$

where $\boldsymbol{F}_{\mathrm{t}-1}$ is a matrix computed at previous sample and $\boldsymbol{F}$ is the sought matrix computed at the current time instant.

As it has been stated in (Horla, 2007a), imposing symmetrical constraints on rates of elements of $\underline{u}_{\mathrm{t}}$ is equivalent to imposing constraints on

$$
\left\|\underline{u}_{t}\right\|_{\max } \leqslant \max _{\underline{x} \in \varepsilon}\left\|\left(\boldsymbol{Y} \boldsymbol{Q}^{-1}-\boldsymbol{F}_{t-1}\right) \underline{x}\right\|_{\max },=\max _{i}\left(\boldsymbol{Y} \boldsymbol{Q}^{-1} \boldsymbol{Y}^{T}-\boldsymbol{F}^{\star}\right)_{i i}
$$

where $\boldsymbol{F}^{*}=\boldsymbol{F}_{t-1} \boldsymbol{Y}^{T}$ should be a symmetrical matrix.

Assuming that $\boldsymbol{F}_{t-1} \boldsymbol{Y}^{T}=\boldsymbol{F}_{t-1} \boldsymbol{Y}_{t-1}^{T}$ hold, one can obtain approximate rough rate constraint as

$$
\left\|\Delta \underline{u}_{t}\right\|_{\max } \leqslant \max _{i}\left(\boldsymbol{Y} \boldsymbol{Q}^{-1} \boldsymbol{Y}^{T}-\boldsymbol{F}_{t-1} \boldsymbol{Y}_{t-1}^{T}\right)_{i i},
$$

or as LMI

$$
\left[\begin{array}{cc}
\boldsymbol{\Lambda}_{\beta}+\boldsymbol{F}_{t-1} \boldsymbol{Y}_{t-1}^{T} & \star \\
\boldsymbol{Y}^{T} & \boldsymbol{Q}
\end{array}\right] \geqslant 0
$$

where $\boldsymbol{\Lambda}_{\beta}=\operatorname{diag}\left\{\beta_{1}^{2}, \ldots, \beta_{m}^{2}\right\}$ is a diagonal matrix comprising squares of rate constraints of $\mathrm{u}_{1, \mathrm{t}}, \ldots, \mathrm{u}_{\mathrm{m}, \mathrm{t}}$ on its diagonal.

The rates of elements of the control vector $\underline{u}_{t}$ are constrained if the above LMI is added to the set of LMIs (30)-(32).

\section{Performance index, evaluation of directional change}

Evaluation of control performance that is coupled with anti-windup compensation requires following indices to be introduced:

$$
\begin{aligned}
& J_{1}=\frac{1}{N} \sum_{i=1}^{p} \sum_{t=1}^{N}\left|r_{i, t}-y_{i, t}\right|, \\
& J_{2}=\frac{1}{N} \sum_{i=1}^{p} \sum_{t=1}^{N}\left(r_{i, t}-y_{i, t}\right)^{2}, \\
& \bar{\varphi}_{1}=\frac{1}{N} \sum_{t=1}^{N}\left|\varphi\left(\underline{v}_{t}\right)-\varphi\left(\underline{u}_{t}\right)\right|\left[{ }^{\circ}\right],
\end{aligned}
$$




$$
\bar{\varphi}_{2}=\frac{1}{N} \sum_{t=1}^{N}\left(\varphi\left(\underline{v}_{t}\right)-\varphi\left(\underline{u}_{t}\right)\right)^{2},
$$

where (40) corresponds to mean absolute tracking error of $p$ outputs, (42) is a mean absolute direction change in between computed and constrained control vector, and $\varphi$ denotes angle measure. In the case of $m=3, \varphi$ corresponds to absolute angle measure (in which case there is no need to decide its direction).

\section{Plant models for simulation studies}

The considered plants are taken with delay $d=1$ and are cross-coupled (values of control offset have been given for reference signals of amplitude 3, where in the case of P3 the last amplitude of reference signal is zero):

- $\quad \mathrm{P} 1(m=2, p=2)$

$$
\boldsymbol{A}=\left[\begin{array}{rr|r}
-0.80 & 0.10 & \boldsymbol{I} \\
-0.40 & 1.00 & \\
\hline 0.49 & 0.10 & \mathbf{0}
\end{array}\right], \boldsymbol{B}=\left[\begin{array}{ll}
1.0 & 0.3 \\
0.5 & 0.8 \\
0.0 & 0.0 \\
0.0 & 0.0
\end{array}\right]
$$

- $\quad \mathrm{P} 2(m=3, p=2)$

$$
\boldsymbol{A}=\left[\begin{array}{rr|r}
-0.80 & 0.10 & \boldsymbol{I} \\
-0.40 & 1.00 & \\
\hline 0.49 & 0.10 & \mathbf{0}
\end{array}\right], \boldsymbol{B}=\left[\begin{array}{lll}
1.0 & 0.2 & 0.3 \\
0.5 & 0.3 & 0.8 \\
0.0 & 0.0 & 0.0 \\
0.0 & 0.0 & 0.0
\end{array}\right]
$$

- $\quad \mathrm{P} 3(m=2, p=3)$

$$
\boldsymbol{A}=\left[\begin{array}{rrr|r}
0.7 & 0.0 & -0.1 & \\
0.1 & 0.8 & -0.2 & \boldsymbol{I} \\
-0.1 & 0.0 & 0.8 & \\
\hline 0.1 & 0.0 & 0.0 & \\
0.0 & -0.1 & 0.0 & \mathbf{0} \\
0.0 & 0.0 & -0.5 &
\end{array}\right], \boldsymbol{B}=\left[\begin{array}{rr}
1.0 & 0.1 \\
0.2 & 1.0 \\
0.5 & -0.1 \\
0.0 & 0.0 \\
0.0 & 0.0 \\
0.0 & 0.0
\end{array}\right]
$$

\section{Simulation studies}

The performed simulations present anti-windup compensation performance for three different plants and hard constraints imposed on the components of the control vector. There are two systems simulated and depicted in the Figure 5, namely systemwith amplitude constraints only and with added rate constraints, clipping only a part of the rates of changes in order to enable a comparison. From Table 1 it is visible that for this case of system with equal number of inputs and outputs, thus possible for dynamic decouplingwith time-varying state-feedback control law, introducing additional constraints causes performance indices to increase (i.e. there is more windup phenomenon in the system) and, simultaneously it causes more severe directional change. 
This is because of the fact that one-step-ahead cannot foresee future changes of reference signals, nor can assure perfect decoupling. Since imposed constraints alter the direction of unconstrained control vector, directional change is necessary to alter the coupling in the system, in order to achieve better control performance.

a)
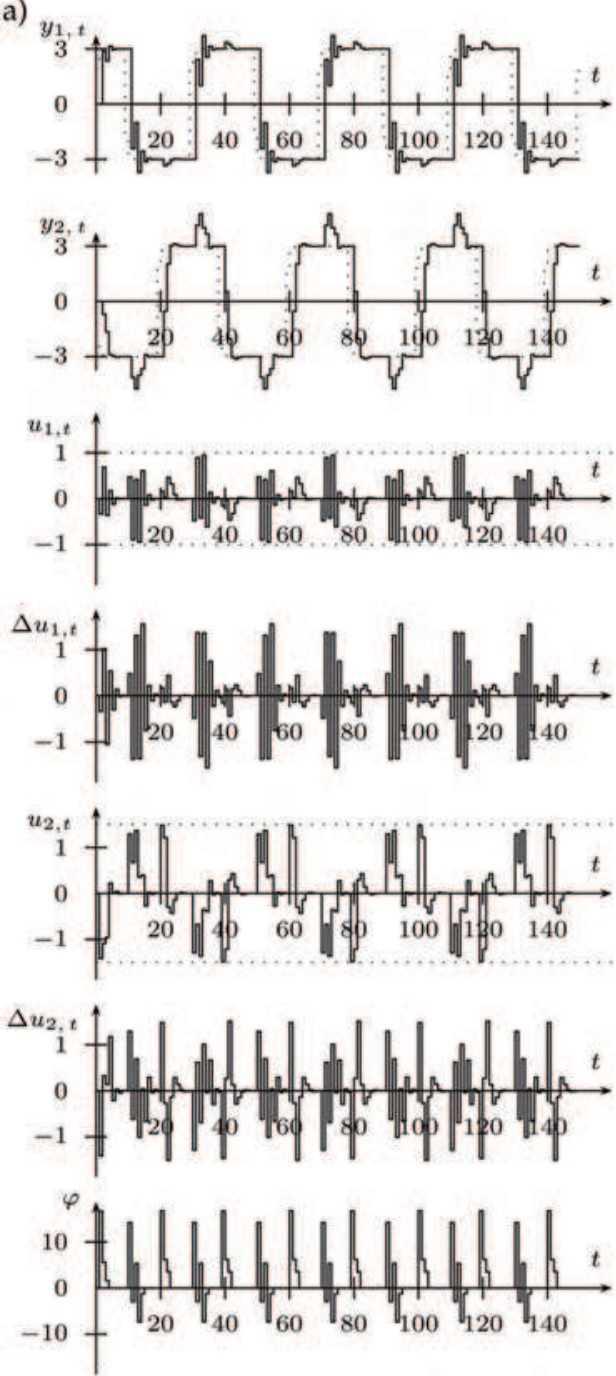

Fig. 5. P1: a) $\alpha_{1}=1.0, \alpha_{2}=1.5, \beta_{1}=\beta_{2}=\infty$, b)
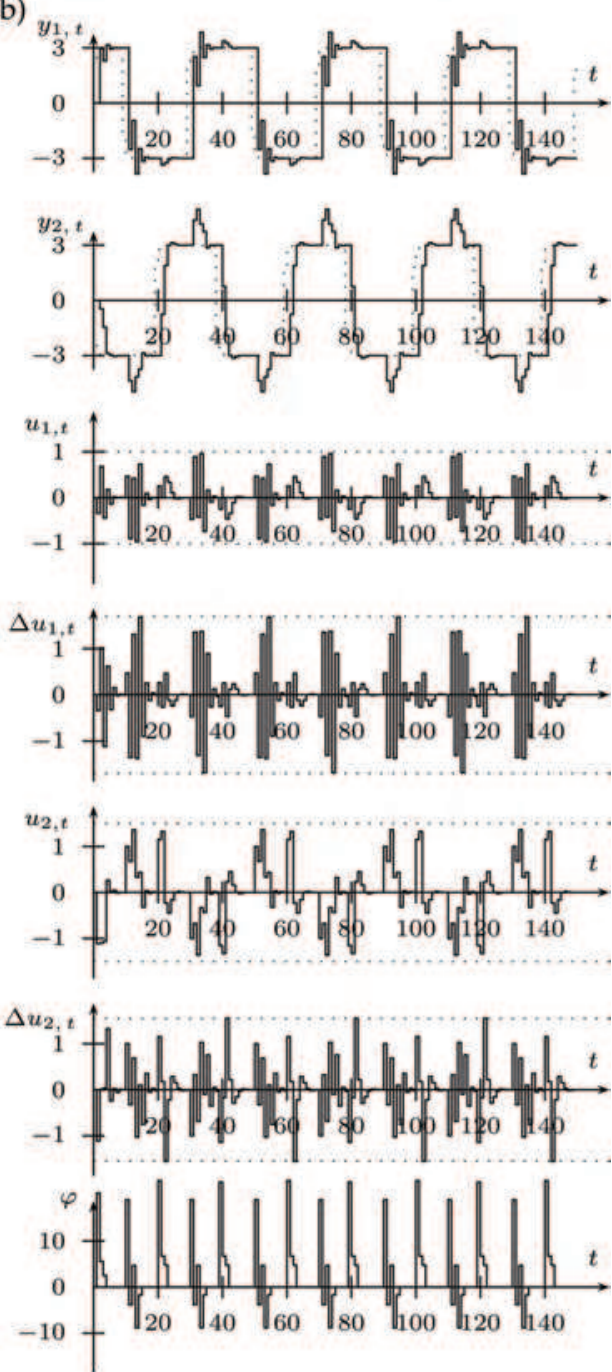

b) $\left.\alpha_{1}=1.0, \alpha_{2}=1.5, \beta_{1}=1.7, \beta_{2}=1.5\right)$ 
a)

$y_{1}$
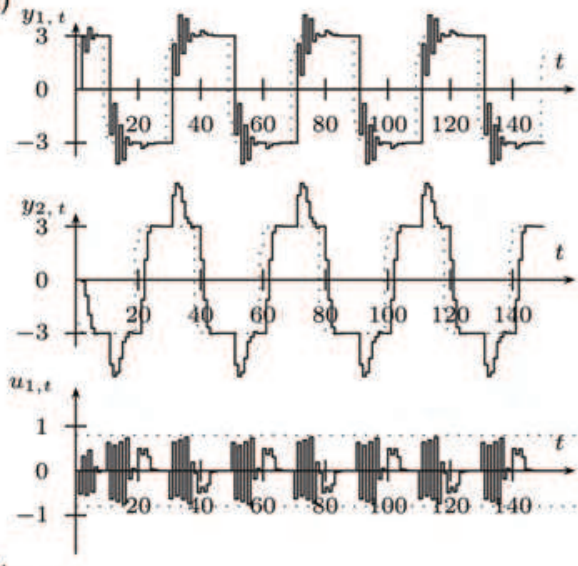

$\Delta u_{1}$,
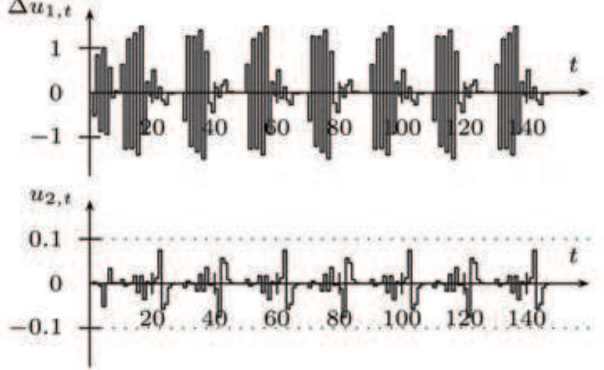

$\Delta u_{2, t}$
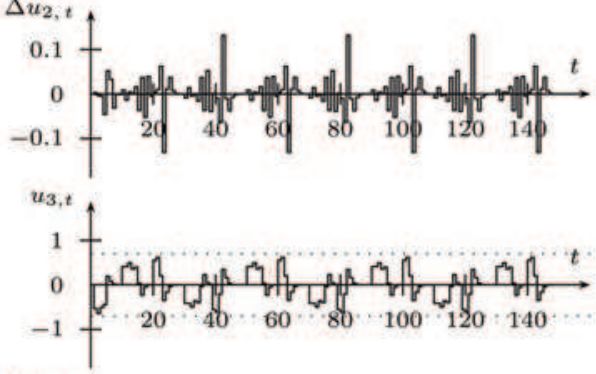

$\Delta u_{3, t}$
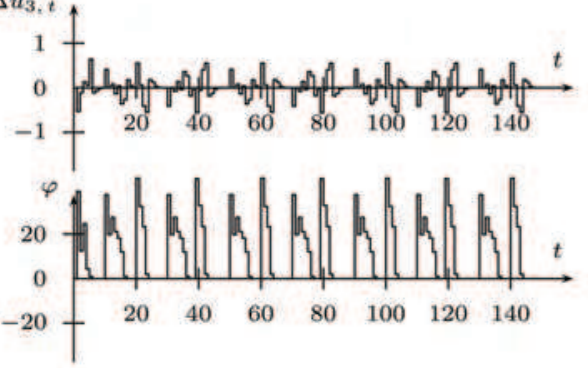

b)
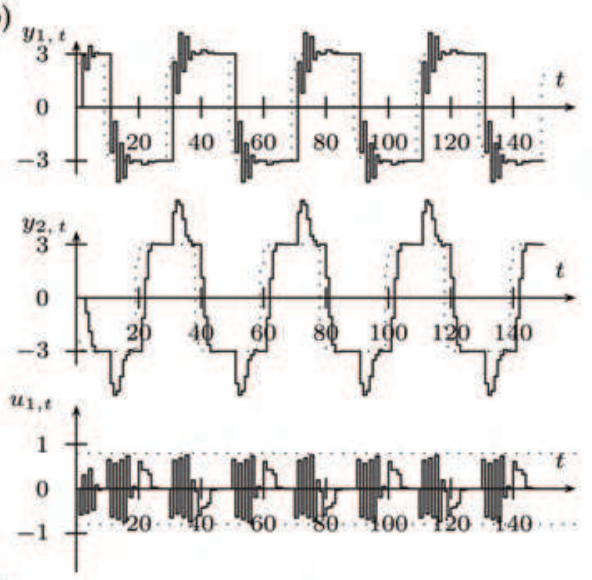

$\Delta u_{1, t}$
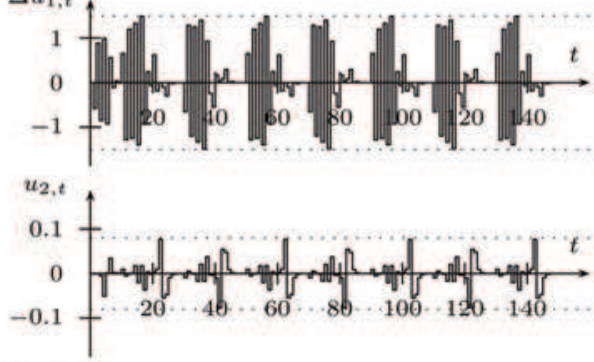

$\Delta u_{2}$,
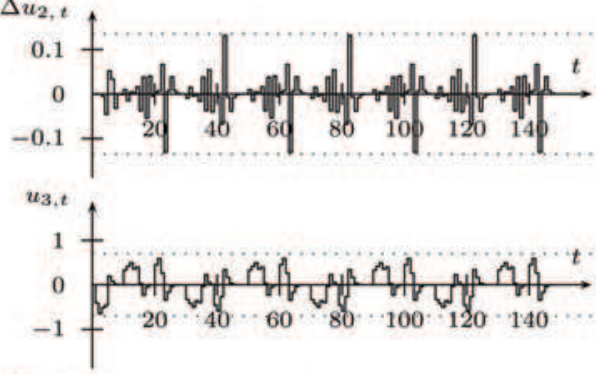

$\Delta u_{3, t}$

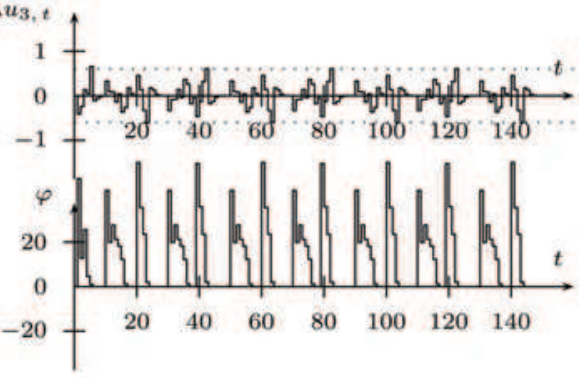

Fig. 6. P2: a) $\alpha_{1}=0.8, \alpha_{2}=0.1, \alpha_{3}=0.7, \beta_{1}=\beta_{2}=\beta_{3}=\infty$, b) $\alpha_{1}=0.8, \alpha_{2}=0.1, \alpha_{3}=0.7, \beta_{1}=1.5$, $\beta_{2}=0.14, \beta_{3}=0.6$ ) 
a)
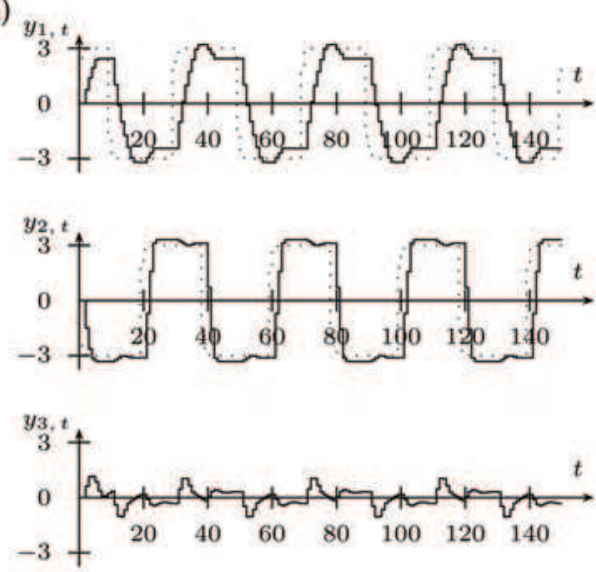

$u_{1,}$

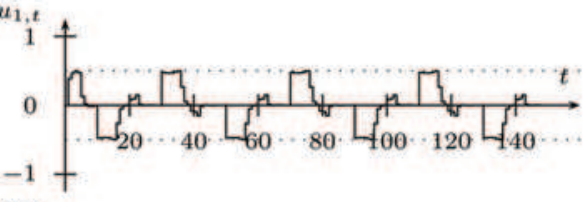

$\Delta u_{1}$

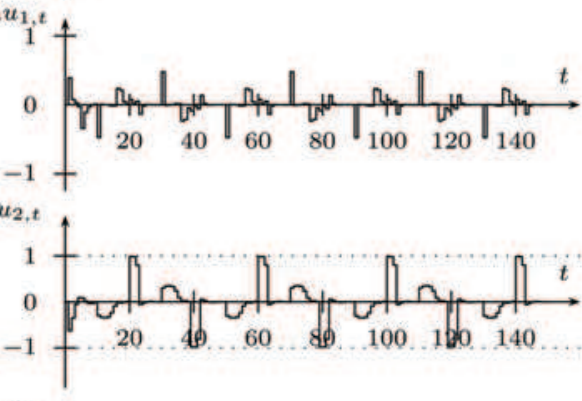

$\Delta u_{2, t}$
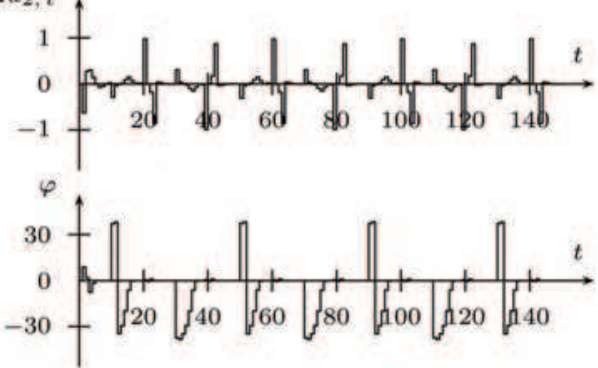

b)
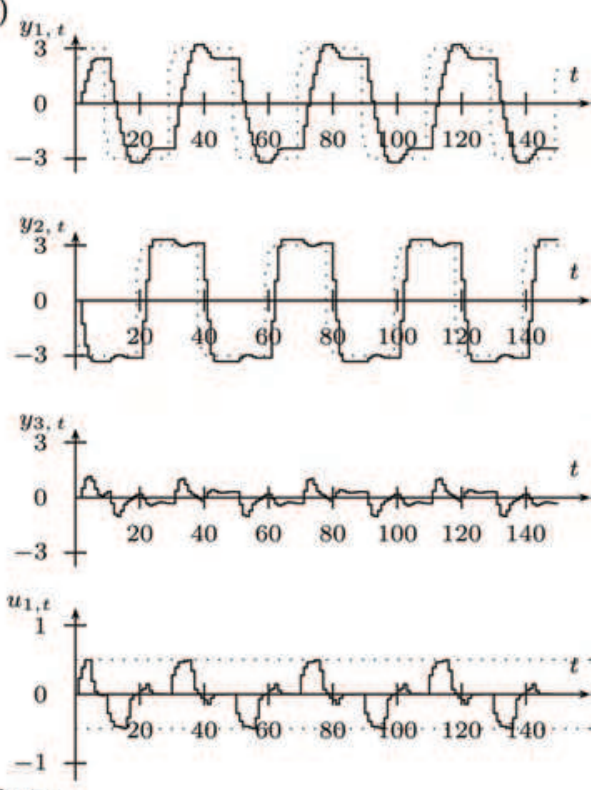

$\Delta u_{1}$

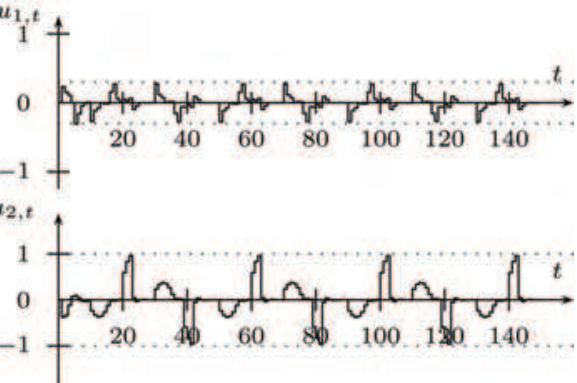

$\Delta u_{2, t}$

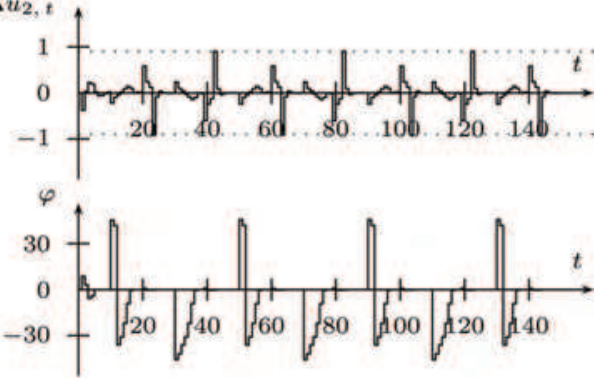

Fig. 7. P3: a) $\alpha_{1}=1.0, \alpha_{2}=1.5, \beta_{1}=\beta_{2}=\infty$, b) $\alpha_{1}=1.0, \alpha_{2}=1.5, \beta_{1}=0.3, \beta_{2}=0.9$ ) 


\begin{tabular}{|c|c|c|c|c|c|c|c|}
\hline & $i=1$ & $i=2$ & \multirow{2}{*}{$\mathrm{P} 2 \mathrm{a}$} & \multirow{2}{*}{\multicolumn{2}{|c|}{$i=1 \quad i=2$}} & P3a & $i=1 \quad i=2$ \\
\hline$J$ & $0-1$ & 5.0601 & & & & $J_{i 1}$ & 1.69473 .4929 \\
\hline & 2.0991 & 5.0001 & $J_{i 1}$ & 2.1408 & 0.3081 & $J_{i 2}$ & 2.24195 .5571 \\
\hline$\frac{J_{i 2}}{I}$ & 2.2919 & 5.9127 & $J_{i 2}$ & $\frac{2.4300}{15708}$ & 7.0549 & $J_{i 3}$ & 0.36130 .2092 \\
\hline$J_{i}$ & 4.3910 & 10.9120 & $J_{i}$ & |4.0700 & 12.00000 & $J_{i}$ & 4.29799 .2592 \\
\hline Plb & 1 & $=2$ & $\mathrm{P} 2 \mathrm{~b}$ & $i=1$ & & P3b & $i=1 \quad i=2$ \\
\hline$J_{i 1}$ & 2.1103 & 5.1055 & $J_{i 1}$ & 2,1386 & 5.3000 & $J_{i 1}$ & 1.71333 .5442 \\
\hline$J_{i 2}$ & 2.3239 & 6.1269 & $J_{i 2}$ & 24353 & 7.1140 & $J_{i 2}$ & 2.21015 .5040 \\
\hline$J_{i}$ & 4.4349 & 112324 & 0,2 & 45730 & 24140 & $J_{i 3}$ & $\begin{array}{lll}0.3548 & 0.1982\end{array}$ \\
\hline & $80.76-12$ & & & | & 12.4140 & $J_{i}$ & 4.27829 .2464 \\
\hline & P1a & $\mathrm{P} 2 \mathrm{a}$ & $\mathrm{P}$ & & P1b & & P3b \\
\hline $\bar{\varphi}_{1}$ & 2.8383 & 11.7238 & 7.9 & & 3.564 & & 8.8754 \\
\hline $\bar{\varphi}_{2}$ & 31.2958 & 47.2096 & 0.0 & & $\bar{\varphi}_{2} \mid 52.969$ & 434 & 2473310.9078 \\
\hline
\end{tabular}

Table 1. Performance indices: a) without, b) with rate constraints

In the case of plant with greater number of control inputs than plant outputs (P2), Figure 6, it is visible that in comparison with P1 the directional change is more severe, because of three components of control vector changing in time. There are more degrees or freedom (ways in which control vector may very in time, it varies in space to be exact, instead as previously in plane), thus it is necessary to alter its direction in order to improve decoupling. Once again performance indexes tend to increase with rate constraints added.

For the last plant considered, P3, it is obvious that since the number of control inputs is deficient in comparison with plant outputs, direction of control vector corresponds to good tracking performance, thus having introduced rate constraints it should not be altered excessively. By comparison of directional change indices and performance indices for P3 it is visible that by introducing rate constraints one can avoid bumps in control signals, what leads to better tracking.

\section{Concluding remarks}

Solving the windup phenomenon problem does not have to mean that constrained control vector is of the same direction as computed control vector if cross-coupling is present in the control system. The presented state-feedback control law by avoiding generation of infeasible control actions compensated windup phenomenon in a priori manner, though as it can be seen, directional change is present in the system in order to assure better decoupling.

On the other hand, avoiding directional change in control enables one to avoid windup phenomenon if and only in the plant is perfectly decoupled or is not coupled at all. The latter due to the constraints is hardly ever met, and if the application of some control law does no require to preserve direction of control vector (what might correspond to, e.g., keeping proportions in between control signals), one should allow directional change to take place. This will both improve anti-windup compensation performance and plant decoupling. 


\section{References}

Albertos, P. and Sala, A. (2004). Multivariable Control Systems. Springer-Verlag, London, United Kingdom.

Boyd, S., Ghaoui, L. E., Feron, E., and Balakrishnan, V. (1994). Linear Matrix Inequalities in System and Control Theory. Society for Industrial and AppliedMathematics, Philadelphia, United States of America, 3rd edition.

Boyd, S. and Vandenberghe, L. (2004). Convex Optimization. Cambridge University Press, United Kingdom.

Doná, J. D., Goodwin, G., and Seron, M. (2000). Anti-windup and model predictive control: Reflections and connections. European Journal of Control, 6(5):455-465.

Horla, D. (2004a). Anti-windup compensators (in Polish). Studies in Automation and Information Technology, 28/29:35-52.

Horla, D. (2004b). Direction alteration of control vector and anti-windup compensation for multivariable systems (in Polish). Studies in Automation and Information Technology, 28/29:53-68.

Horla, D. (2006a). LMI-based multivariable adaptive predictive controller with anti-windup compensator. In Proceedings of the 12th IEEE International Conference MMAR, pages 459- 462, Miedzyzdroje.

Horla, D. (2006b). Multivariable state-feedback control with windup phenomenon avoidance. In Proceedings of the 18th ICSS, pages 143-146, Coventry.

Horla, D. (2006c). Standard vs. LMI approach to a convex optimisation problem in multivariable predictive control task with a priori anti-windup compensator. In Proceedings of the 18th ICSS, pages 147-152, Coventry.

Horla, D. (2007a). Close-to-optimal adaptive multivariable state-feedback controller with a priori anti-windup compensator. In Proceedings of the 13th IEEE IFAC International Conference on Methods and Models in Automation and Robotics, pages 363-368, Szczecin.

Horla, D. (2007b). Directional change and windup phenomenon. In Proceedings of the 4th IFAC International Conference on Informatics in Control Automation and Robotics, pages CD-ROM, Angers, France.

Horla, D. (2007c). Directional change for rate-constrained systems with anti-windup compensation. In Proceedings of the 16th International Conference on Systems Science, volume 1, pages 122-131, Wrocław.

Horla, D. (2007d). Optimised conditioning technique for a priori anti-windup compensation. In Proceedings of the 16th International Conference on Systems Science, pages 132-139, Wrocław, Poland.

Maciejowski, J. (1989). Multivariable Feedback Design. Addison-Wesley Publishing Company, Cambridge, United Kingdom.

Öhr, J. (2003). Anti-windup and Control of Systems withMultiple Input Saturations: Tools, Solution and Case Studies. PhD thesis, Uppsala University, Uppsala, Sweden.

Peng, Y., Vran`ci'c, D., Hanus, R., and Weller, S. (1998). Anti-windup designs for multivariable controllers. Automatica, 34(12):1559-1565.

Walgama, K. and Sternby, J. (1993). Contidioning technique for multiinput multioutput processes with input saturation. IEE Proceedings-D, 140(4):231-241. 


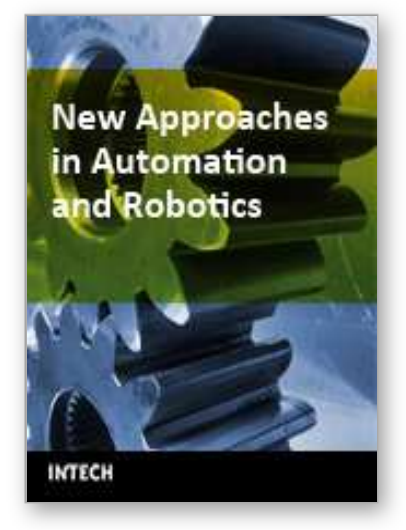

\author{
New Approaches in Automation and Robotics \\ Edited by Harald Aschemann
}

ISBN 978-3-902613-26-4

Hard cover, 392 pages

Publisher I-Tech Education and Publishing

Published online 01, May, 2008

Published in print edition May, 2008

The book New Approaches in Automation and Robotics offers in 22 chapters a collection of recent developments in automation, robotics as well as control theory. It is dedicated to researchers in science and industry, students, and practicing engineers, who wish to update and enhance their knowledge on modern methods and innovative applications. The authors and editor of this book wish to motivate people, especially under-graduate students, to get involved with the interesting field of robotics and mechatronics. We hope that the ideas and concepts presented in this book are useful for your own work and could contribute to problem solving in similar applications as well. It is clear, however, that the wide area of automation and robotics can only be highlighted at several spots but not completely covered by a single book.

\title{
How to reference
}

In order to correctly reference this scholarly work, feel free to copy and paste the following:

Dariusz Horla (2008). Directional Change Issues in Multivariable State-Feedback Control, New Approaches in Automation and Robotics, Harald Aschemann (Ed.), ISBN: 978-3-902613-26-4, InTech, Available from: http://www.intechopen.com/books/new_approaches_in_automation_and_robotics/directional_change_issues_i n_multivariable_state-feedback_control

\section{INTECH}

open science | open minds

\section{InTech Europe}

University Campus STeP Ri

Slavka Krautzeka 83/A

51000 Rijeka, Croatia

Phone: +385 (51) 770447

Fax: +385 (51) 686166

www.intechopen.com

\section{InTech China}

Unit 405, Office Block, Hotel Equatorial Shanghai

No.65, Yan An Road (West), Shanghai, 200040, China

中国上海市延安西路65号上海国际贵都大饭店办公楼 405 单元

Phone: +86-21-62489820

Fax: +86-21-62489821 
(C) 2008 The Author(s). Licensee IntechOpen. This chapter is distributed under the terms of the Creative Commons Attribution-NonCommercialShareAlike-3.0 License, which permits use, distribution and reproduction for non-commercial purposes, provided the original is properly cited and derivative works building on this content are distributed under the same license. 